\title{
BLOCK NORMAL MATRICES AND GERSHGORIN-TYPE DISCS*
}

\author{
JAKUB KIERZKOWSKI ${ }^{\dagger}$ AND ALICJA SMOKTUNOWICZ ${ }^{\dagger}$
}

\begin{abstract}
The block analogues of the theorems on inclusion regions for the eigenvalues of normal matrices are given. By an inclusion region for a given matrix $A$ we mean a region of the complex plane that contains at least one of the eigenvalues of $A$. Some nonsingularity results for partitioned matrices are also presented.
\end{abstract}

Key words. Normal matrices, Block matrices, Eigenvalues, Gershgorin's discs, Nonsingular matrices, Hermitian positive definite matrices, Strong square-sum criterion.

AMS subject classifications. 15A12, 15A 18 .

1. Introduction. The purpose of this paper is to derive new inclusion regions of Gershgorin type for partitioned normal matrices; see Theorems 2.7-2.8. Theorem 2.7 improves the results of Meyer and Veselić; see [5, p. 436]. We also give the block versions of nonsingularity results; see Theorem 2.9. Theorem 2.9 is an analogue of Varga's Theorem 6.2 for strictly block diagonally dominant matrices; see [11, p. 157]. We review some of the standard facts on the location of eigenvalues of block matrices which are useful in error analysis of numerical algorithms and in study convergence of block iterative methods for solving linear systems of equations.

The set of all $n$-by- $n$ matrices over $\mathbb{C}$ is denoted by $\mathcal{M}_{n}$. We recall that a normal matrix $A \in \mathcal{M}_{n}$ is any matrix satisfying $A A^{*}=A^{*} A$, where $A^{*}$ is the conjugate transpose of a matrix $A$. Normal matrices $A$ can be Hermitian $\left(A^{*}=A\right)$, skewHermitian $\left(A^{*}=-A\right)$, and unitary $\left(A^{*} A=I_{n}\right)$, where $I_{n}$ is the $n \times n$ identity matrix. We consider only the spectral matrix norm and the second vector norm. Throughout the paper, $\sigma(A)$ denotes the set of all eigenvalues of $A, \rho(A)=\max \{|\lambda|: \lambda \in \sigma(A)\}$ is the spectral radius of $A$ and $R(x, A)=x^{*} A x$ is the Rayleigh quotient of $x \in \mathbb{C}^{n}$ with $\|x\|_{2}=1$.

First, we review some important facts on the location of eigenvalues of matrices.

Theorem 1.1. (Gershgorin, 1931) The eigenvalues of $A \in \mathcal{M}_{n}$ lie in the union

${ }^{*}$ Received by the editors on July 14, 2007. Accepted for publication on October 5, 2011. Handling Editor: Richard Brualdi.

${ }^{\dagger}$ Faculty of Mathematics and Information Science, Warsaw University of Technology, Pl. Politechniki 1, Warsaw, 00-661 Poland (J.Kierzkowski@mini.pw.edu.pl, A.Smoktunowicz@mini.pw.edu.pl). 
$\bigcup_{i=1}^{n} G_{i}$ of the $n$ discs

$$
G_{i}=\left\{z \in \mathbb{C}: \quad\left|z-a_{i i}\right| \leq r_{i}(A)\right\}
$$

where $r_{i}(A)$ denotes the deleted absolute row sums of $A$, i.e.,

$$
r_{i}(A)=\sum_{j \neq i}\left|a_{i j}\right| .
$$

However, some Gershgorin's discs may contain no eigenvalues. In case of normal matrices, such situation does not occur.

Theorem 1.2. ([7]) Let $A \in \mathcal{M}_{n}$ be normal. Then each of the discs

$$
E_{i}=\left\{z \in \mathbb{C}: \quad\left|z-a_{i i}\right| \leq s_{i}(A)\right\},
$$

where

$$
s_{i}(A)=\sqrt{\sum_{j \neq i}\left|a_{i j}\right|^{2}},
$$

must contain an eigenvalue of $A$.

Using the Cauchy-Schwartz inequality we get

$$
s_{i}(A) \leq r_{i}(A) \leq \sqrt{n-1} s_{i}(A), \quad i=1, \ldots, n .
$$

Thus, $E_{i} \subseteq G_{i}$ for all $i=1, \ldots, n$, so the Euclidean discs $E_{i}$ are smaller than the Gershgorin discs $G_{i}$. Unfortunately, some eigenvalues of the normal matrix $A$ can be located outside the set $\bigcup_{i=1}^{n} E_{i}$.

TheOREM 1.3. For any $\lambda \in \mathbb{C}$ and $n>2$, there exists a normal matrix $A \in \mathcal{M}_{n}$ such that $\lambda$ is an eigenvalue of $A$ and

$$
\left|\lambda-a_{i i}\right|>s_{i}(A) \quad(i=1, \ldots, n) .
$$

Proof. Let $A=(\lambda-n) I_{n}+J$, where $J$ has each entry equal to 1 . It is easily seen that $\sigma(J)=\{0, n\}$. Therefore, $\sigma(A)=\{\lambda, \lambda-n\}$. From this it follows that $\left|\lambda-a_{i i}\right|=n-1>s_{i}(A)=\sqrt{n-1}$ for all $i$. This completes the proof. $\square$

It is well-known that if $A \in \mathcal{M}_{n}$ is strictly diagonally dominant (i.e., $A$ satisfies the strong row-sum criterion: $\left|a_{i i}\right|>r_{i}(A)$ for all $\left.i=1, \ldots, n\right)$, then $A$ is nonsingular; see, for example, [1]. Some other interesting nonsingularity results were given in [1]. Now we present a new nonsingularity result involving the radii $s_{i}(A)$. 
THEOREM 1.4. If $A \in \mathcal{M}_{n}$ satisfies the strong square-sum criterion, i.e.,

$$
\omega(A)=\sum_{i=1}^{n} \frac{s_{i}^{2}(A)}{\left|a_{i i}\right|^{2}}<1,
$$

then $A$ is nonsingular.

Moreover, if $A$, satisfying the strong square-sum criterion, is Hermitian and all of its diagonal elements $a_{i i}$ are positive, then $A$ is positive definite.

We give a proof of this theorem in Section 2; see Theorem 2.9. It is known that the strong square-sum criterion and the strong row-sum criterion are sufficient conditions for the convergence of the Jacobi method for solving a linear system of equations $A x=b$; see [4, p. 359]. Notice that these criteria are not equivalent.

EXAMPLE 1.5. Let

$$
A_{1}=\left[\begin{array}{cccc}
88 & 7 & 12 & 17 \\
7 & 78 & 17 & 12 \\
12 & 17 & 68 & 27 \\
17 & 22 & 27 & 58
\end{array}\right], \quad A_{2}=\left[\begin{array}{rrrr}
14 & -6 & 4 & -1 \\
-6 & 24 & -11 & 3 \\
4 & -11 & 20 & -3 \\
-1 & 3 & -3 & 11
\end{array}\right]
$$

It is easily seen that $A_{1}$ is not strictly diagonally dominant but satisfies the strong square-sum criterion with $\omega\left(A_{1}\right) \approx 0.8393$. On the other hand, $A_{2}$ does not satisfy the strong square-sum criterion, since $\omega\left(A_{2}\right) \approx 1.0806$. However, $A_{2}$ is strictly diagonally dominant.

We extend these results to partitioned matrices in Section 2. Some of them can be derived from the following residual theorem; see, e.g., [4, p. 104].

Theorem 1.6. If $A \in \mathcal{M}_{n}$ is a normal matrix with eigenvalues $\lambda_{1}, \ldots, \lambda_{n}$, then for any $\alpha \in \mathbb{C}$ and any vector $x \in \mathbb{C}^{n}$ with $\|x\|_{2}=1$

$$
\min _{i=1, \ldots, n}\left|\lambda_{i}-\alpha\right| \leq\|A x-\alpha x\|_{2} .
$$

To illustrate the theory, we present numerical tests in Section 3.

2. Inclusion theorems for block matrices. Let the matrix $A \in \mathcal{M}_{n}$ be partitioned into $q \times q$ blocks

$$
A=\left[A_{i j}\right]=\left[\begin{array}{cccc}
A_{11} & A_{12} & \cdots & A_{1 q} \\
A_{21} & A_{22} & \cdots & A_{2 q} \\
\vdots & \vdots & \ddots & \vdots \\
A_{q 1} & A_{q 2} & \cdots & A_{q q}
\end{array}\right]
$$


where $A_{i, j} \in \mathbb{C}^{n_{i}, n_{j}}$ is the $(i, j)$ block of $A,\left\{n_{1}, \ldots, n_{q}\right\}$ is a given set of positive integers, and $n_{1}+\cdots+n_{q}=n$.

First, we recall very useful estimations of the eigenvalues of partitioned matrices, frequently used in error analysis of numerical algorithms and in the study of convergence of iterative methods for solving large linear systems of equations.

TheOREM 2.1. Assume that $A \in \mathcal{M}_{n}$ is partitioned as in (2.1). If $\lambda \in \sigma(A)$, then

$$
|\lambda| \leq \rho(\mu(A)) \leq\|\mu(A)\|_{2},
$$

where $\mu(A)$ is a matricial norm of $A$, defined as

$$
\mu(A)=\left[\begin{array}{cccc}
\left\|A_{11}\right\|_{2} & \left\|A_{12}\right\|_{2} & \cdots & \left\|A_{1 q}\right\|_{2} \\
\left\|A_{21}\right\|_{2} & \left\|A_{22}\right\|_{2} & \cdots & \left\|A_{2 q}\right\|_{2} \\
\vdots & \vdots & \ddots & \vdots \\
\left\|A_{q 1}\right\|_{2} & \left\|A_{q 2}\right\|_{2} & \cdots & \left\|A_{q q}\right\|_{2}
\end{array}\right] .
$$

Theorem 2.1 is a generalization of the Perron-Frobenius inequality and was first proved by Ostrowski; see [2], [6] and [10].

If, additionally, the matrix $A$ is normal, one can estimate real and imaginary parts of eigenvalues of $A$ in terms of eigenvalues of diagonal blocks $A_{k k}$; see also [8] where interesting analogue of min-max theorem for normal matrices was presented.

TheOREM 2.2. Assume that $A \in \mathcal{M}_{n}$ is normal and partitioned as in (2.1). Let $\sigma(A)=\left\{\lambda_{1}, \lambda_{2}, \ldots, \lambda_{n}\right\}$. Then, for any $k \in\{1,2, \ldots, q\}$ and any $\alpha \in \sigma\left(A_{k k}\right)$, the following inequalities hold

$$
\min _{i=1, \ldots, n} \mathfrak{R} e \lambda_{i} \leq \mathfrak{R e} \alpha \leq \max _{i=1, \ldots, n} \mathfrak{R} e \lambda_{i}
$$

and

$$
\min _{i=1, \ldots, n} \mathfrak{I} m \lambda_{i} \leq \Im m \alpha \leq \max _{i=1, \ldots, n} \Im m \lambda_{i} .
$$

Proof. By the normality of $A$, there exist a unitary matrix $U$ and a diagonal matrix $D$ such that $A=U D U^{*}$ with $D=\operatorname{diag}\left(\lambda_{1}, \lambda_{2}, \ldots, \lambda_{n}\right)$. Let $y=U^{*} x$, where $x \in \mathbb{C}^{n}$ and $\|x\|_{2}=1$. Then $\|y\|_{2}=1$ and the Rayleigh quotient $R(x, A)=x^{*} A x$ can be written as $R(x, A)=R(y, D)=\sum_{i=1}^{n} \lambda_{i}\left|y_{i}\right|^{2}$. From this we obtain

$$
\min _{i=1, \ldots, n} \mathfrak{R e} \lambda_{i} \leq \mathfrak{R e} R(x, A) \leq \max _{i=1, \ldots, n} \mathfrak{R e} \lambda_{i}
$$


and

$$
\min _{i=1, \ldots, n} \mathfrak{I m} \lambda_{i} \leq \mathfrak{I m} R(x, A) \leq \max _{i=1, \ldots, n} \mathfrak{I m} \lambda_{i}
$$

Assume now that $u$ is an eigenvector of $A_{k k}$ such that $\|u\|_{2}=1$ and $A_{k k} u=\alpha u$. Define $x=\left[x_{1}^{T}, x_{2}^{T}, \ldots, x_{q}^{T}\right]^{T}$, where $x_{k} \in \mathbb{C}^{\mathrm{n}_{\mathrm{k}}}$ be such that $x_{i}=0$ for $i \neq k$ and $x_{k}=u$. Then $R(x, A)=R\left(u, A_{k k}\right)=\alpha$. From this and (2.6)-(2.7), we get $(2.4)-(2.5)$.

Notice that normality of $A$ in Theorem 2.2 is crucial; see the following easy example.

EXAMPLE 2.3. Let

$$
A=\left[\begin{array}{cc}
1 & 2 \\
-2 & 3
\end{array}\right]
$$

Then $A$ is not normal and the eigenvalues of $A$ are $\lambda_{1,2}=2 \pm i \sqrt{3}$. We see that (2.4) is not valid in this case.

Feingold and Varga proved the following Gershgorin-type theorem for partitioned matrices; see [3] and [11, pp. 157-159].

Theorem 2.4. Assume that $A \in \mathcal{M}_{n}$ is partitioned as in (2.1). If $\lambda \in \sigma(A)$ and $\lambda \notin \sigma\left(A_{k k}\right)$ for all $k$, then there exists $i \in\{1, \ldots, q\}$ such that

$$
\frac{1}{\left\|\left(\lambda I-A_{i i}\right)^{-1}\right\|_{2}} \leq \sum_{j \neq i}\left\|A_{i j}\right\|_{2} .
$$

However, it is difficult to compute this inclusion region in practice. If all diagonal blocks $A_{k k}$ are normal, then the following theorem holds; see [5].

Corollary 2.5. Assume that $A \in \mathcal{M}_{n}$ is partitioned as in (2.1). Suppose that all diagonal blocks $A_{k k}$ of $A$ are normal. If $\lambda$ is an eigenvalue of $A$, then there exists $i \in\{1,2, \ldots, q\}$ such that

$$
\min _{\alpha \in \sigma\left(A_{i i}\right)}|\lambda-\alpha| \leq \sum_{j=1, j \neq i}^{q}\left\|A_{i j}\right\|_{2} .
$$

In [5], Meyer and Veselić obtained the following generalization of Theorem 1.2.

TheOREM 2.6. Assume that a normal matrix $A \in \mathcal{M}_{n}$ is partitioned as in (2.1). Let

$$
\hat{S}_{i}(A)=\sqrt{\sum_{j=1, j \neq i}^{q}\left\|A_{j i}\right\|_{2}^{2}} \quad(i=1, \ldots, q)
$$


Then each disc of the form $|z-\alpha| \leq \hat{S}_{i}(A)$, where $\alpha$ is an eigenvalue of $A_{i i}$, contains at least one eigenvalue of $A$.

Now we show that in $(2.10)$ one can replace $\hat{S}_{i}(A)$ by smaller radii $S_{i}(A)$.

TheOREM 2.7. Assume that a normal matrix $A \in \mathcal{M}_{n}$ is partitioned as in (2.1). Let

$$
S_{i}(A)=\left\|\left[A_{1 i}^{T}, A_{2 i}^{T}, \ldots, A_{i-1, i}^{T}, A_{i+1, i}^{T}, \ldots, A_{q i}^{T}\right]\right\|_{2} \quad(i=1, \ldots, q) .
$$

Then each disc of the form $|z-\alpha| \leq S_{i}(A)$, where $\alpha$ is an eigenvalue of $A_{i i}$, contains at least one eigenvalue of $A$.

Proof. Let $\alpha$ be an eigenvalue of $A_{i i}$. Then there exists a vector $u \in \mathbb{C}^{\mathrm{n}_{\mathrm{i}}}$ such that $A_{i i} u=\alpha u$ and $\|u\|_{2}=1$.

Define $x=\left[x_{1}^{T}, x_{2}^{T}, \ldots, x_{q}^{T}\right]^{T}$, where $x_{k} \in \mathbb{C}^{\mathrm{n}_{\mathrm{k}}}$ be such that $x_{k}=0$ for $k \neq i$ and $x_{i}=u$. Then

$$
A x-\alpha x=\left[\begin{array}{c}
A_{1 i} u \\
A_{2 i} u \\
\ldots \\
A_{i-1, i} u \\
A_{i i} u-\alpha u \\
A_{i+1, i} u \\
\ldots \\
A_{q i} u
\end{array}\right]=\left[\begin{array}{c}
A_{1 i} \\
A_{2 i} \\
\ldots \\
A_{i-1, i} \\
0 \\
A_{i+1, i} \\
\cdots \\
A_{q i}
\end{array}\right] u .
$$

Taking norms, we get the desired inequality. We have

$$
\|A x-\alpha x\|_{2} \leq\left\|\left[A_{1 i}^{T}, A_{2 i}{ }^{T}, \ldots, A_{i-1, i}^{T}, 0, A_{i+1, i}^{T}, \ldots, A_{q i}^{T}\right]^{T}\right\|_{2}\|u\|_{2} .
$$

Thus,

$$
\|A x-\alpha x\|_{2} \leq S_{i}(A)\|u\|_{2}=S_{i}(A)
$$

From this and Theorem 1.6, we conclude that

$$
\min _{i=1, \ldots, n}\left|\lambda_{i}-\alpha\right| \leq\|A x-\alpha x\|_{2} \leq S_{i}(A) .
$$

This finishes the proof.

Notice that by Ostrowski's theorem we have $S_{i}(A) \leq \hat{S}_{i}(A)$ for all $i=1, \ldots, q$.

Now we give a block analogue of the inequality involving diagonal elements and eigenvalues of $A$; see [9] for unpartitioned case. 
Theorem 2.8. Assume that $A \in \mathcal{M}_{n}$ is partitioned as in (2.1) and define

$$
R_{i}(A)=\sqrt{\sum_{j=1, j \neq i}^{q}\left\|A_{i j}\right\|_{2}^{2}} \quad(i=1, \ldots, q) .
$$

Then each eigenvalue $\lambda$ of $A$ such that $\lambda \notin \sigma\left(A_{i i}\right)$ for all $i=1, \ldots, q$, satisfies

$$
\sum_{i=1}^{q}\left\|\left(\lambda I_{n_{i}}-A_{i i}\right)^{-1}\right\|_{2}^{2} R_{i}^{2}(A) \geq \frac{q}{q-1} .
$$

Proof. Let $\lambda$ be an eigenvalue of $A$, and suppose $A x=\lambda x$ with $\|x\|_{2}=1$. Let $x=\left[x_{1}^{T}, x_{2}^{T}, \ldots, x_{q}^{T}\right]^{T}$, where $x_{i} \in \mathbb{C}^{\mathrm{n}_{\mathrm{i}}}$.

We have

$$
\sum_{j=1}^{q} A_{i j} x_{j}=\lambda x_{i}, \quad i=1, \ldots, q
$$

and thus,

$$
\left(\lambda I_{n_{i}}-A_{i i}\right) x_{i}=\sum_{j=1, j \neq i}^{q} A_{i j} x_{j}, \quad i=1, \ldots, q .
$$

Since $\lambda \notin \sigma\left(A_{i i}\right)$, all matrices $\lambda I_{n_{i}}-A_{i i}$ are nonsingular. From this, it follows that

$$
x_{i}=\left(\lambda I_{n_{i}}-A_{i i}\right)^{-1} \sum_{j=1, j \neq i}^{q} A_{i j} x_{j}, \quad i=1, \ldots, q .
$$

Taking norms, we obtain

$$
\left\|x_{i}\right\|_{2} \leq\left\|\left(\lambda I_{n_{i}}-A_{i i}\right)^{-1}\right\|_{2} \sum_{j=1, j \neq i}^{q}\left\|A_{i j}\right\|_{2}\left\|x_{j}\right\|_{2} .
$$

By the Cauchy-Schwartz inequality, we have

$$
\left(\sum_{j=1, j \neq i}^{q}\left\|A_{i j}\right\|_{2}\left\|x_{j}\right\|_{2}\right)^{2} \leq \sum_{j=1, j \neq i}^{q}\left\|A_{i j}\right\|_{2}^{2} \sum_{j=1, j \neq i}^{q}\left\|x_{j}\right\|_{2}^{2}=R_{i}^{2}(A) \sum_{j=1, j \neq i}^{q}\left\|x_{j}\right\|_{2}^{2},
$$

so we can rewrite $(2.14)$ as follows

$$
\left\|x_{i}\right\|_{2}^{2} \leq\left\|\left(\lambda I_{n_{i}}-A_{i i}\right)^{-1}\right\|_{2}^{2} R_{i}^{2}(A) \sum_{j=1, j \neq i}^{q}\left\|x_{j}\right\|_{2}^{2}, \quad i=1, \ldots, q .
$$


For simplicity of notation, let $b_{i}$ stand for

$$
b_{i}=\sum_{j=1, j \neq i}^{q}\left\|x_{j}\right\|_{2}^{2} .
$$

Since $1=\|x\|_{2}^{2}=\left\|x_{1}\right\|_{2}^{2}+\cdots+\left\|x_{q}\right\|_{2}^{2}$, the elements we have just defined satisfy

$$
b_{i}=1-\left\|x_{i}\right\|_{2}^{2}
$$

and

$$
\sum_{i=1}^{q} b_{i}=\sum_{i=1}^{q}\left(1-\left\|x_{i}\right\|_{2}^{2}\right)=q-\sum_{i=1}^{q}\left\|x_{i}\right\|_{2}^{2}=q-1 .
$$

Thus, we can rewrite $(2.15)$ as follows

$$
\left\|x_{i}\right\|_{2}^{2} \leq\left\|\left(\lambda I_{n_{i}}-A_{i i}\right)^{-1}\right\|_{2}^{2} R_{i}^{2}(A) \quad b_{i}, \quad i=1, \ldots, q
$$

Notice that by the assumptions of our theorem, all $b_{i}$ are positive. From this and (2.17)-(2.19), we get

$$
\begin{gathered}
\sum_{i=1}^{q}\left\|\left(\lambda I_{n_{i}}-A_{i i}\right)^{-1}\right\|_{2}^{2} \quad R_{i}^{2}(A) \geq \sum_{i=1}^{q} \frac{\left\|x_{i}\right\|_{2}^{2}}{b_{i}}= \\
=\sum_{i=1}^{q} \frac{1-b_{i}}{b_{i}}=\sum_{i=1}^{q}\left(\frac{1}{b_{i}}-1\right)=\sum_{i=1}^{q} \frac{1}{b_{i}}-q .
\end{gathered}
$$

By an application of the harmonic-arithmetic mean inequality and from (2.18) we obtain

$$
\sum_{i=1}^{q} \frac{1}{b_{i}} \geq \frac{q^{2}}{\sum_{i=1}^{q} b_{i}}=\frac{q^{2}}{q-1}
$$

This together with (2.20) gives the desired inequality (2.13).

As a corollary we obtain the following generalization of Theorem 1.4.

TheOREM 2.9. If $A \in \mathcal{M}_{n}$ satisfies the strong block square-sum criterion, i.e.,

$$
\sum_{i=1}^{q}\left\|A_{i i}^{-1}\right\|_{2}^{2} \quad R_{i}^{2}(A)<1
$$

then $A$ is nonsingular.

Moreover, if $A$ is Hermitian, satisfies the strong square-sum criterion and all diagonal blocks $A_{i i}$ are positive definite, then $A$ is positive definite. 
Proof. Let us first prove nonsingularity of $A$. On the contrary, suppose that $A$ is singular. Then there is an eigenvalue $\lambda=0$ of $A$. From Theorem 2.8, taking $\lambda=0$ in (2.13), we deduce that $q /(q-1)<1$, which is impossible.

Now assume that $A$ is Hermitian, satisfies the strong square-sum criterion and all diagonal blocks $A_{i i}$ are positive definite. Since $A$ is Hermitian, all its eigenvalues are real. It is sufficient to prove that they are positive. Conversely, suppose that there exists a negative eigenvalue $\hat{\lambda}$ of $A$. By assumption, all matrices $A_{i i}$ are Hermitian and positive definite, so $\hat{\lambda} \notin \sigma\left(A_{i i}\right)$. Since $\hat{\lambda}$ is negative, we conclude that

$$
\left\|\left(\hat{\lambda} I_{n_{i}}-A_{i i}\right)^{-1}\right\|_{2}<\left\|A_{i i}{ }^{-1}\right\|_{2}, \quad i=1, \ldots, q .
$$

By Theorem 2.8, we get

$$
\sum_{i=1}^{q}\left\|A_{i i}^{-1}\right\|_{2}^{2} R_{i}^{2}(A) \geq \sum_{i=1}^{q}\left\|\left(\hat{\lambda} I_{n_{i}}-A_{i i}\right)^{-1}\right\|_{2}^{2} \quad R_{i}^{2}(A) \geq \frac{q}{q-1},
$$

but this contradicts our assumption (2.21). This finishes the proof. $\square$

REMARK 2.10.

- Theorem 2.9 is an analogue of Varga's Theorem 6.2 for strictly block diagonally dominant matrices; see [11, p. 157].

- It is clear that using $A^{T}$ instead of $A$ in the above theorems a new set of the radii can be obtained.

- It is easy to prove, by Ostrowski's theorem, that the strong block square-sum criterion (2.21) is sufficient condition for the convergence of the block Jacobi method for solving a linear system of equations $A x=b$.

3. Computational examples. Numerical tests were given in MATLAB, version 6.5.0.180913a (R13) with unit roundoff $\epsilon \approx 2.2 \cdot 10^{-16}$ in IEEE double precision.

The eigenvalues of $A$ were plotted as crosses 'x'.

EXAMPLE 3.1. Let

$$
A=\left[\begin{array}{ccccccc}
611 & 0 & 0 & 407 & 0 & 0 & -49 \\
0 & 899 & 113 & -192 & 0 & 0 & -8 \\
0 & 113 & 899 & 196 & 0 & 0 & 8 \\
407 & -192 & 196 & 611 & 0 & 0 & 59 \\
0 & 0 & 0 & 0 & 411 & -599 & 208 \\
0 & 0 & 0 & 0 & -599 & 411 & 208 \\
-49 & -8 & 8 & 59 & 208 & 208 & 99
\end{array}\right] .
$$

Then $A$ is normal and $\sigma(A) \approx\{-374.7,122.4,302.9,729.5,1010.0,1012.0,1139.0\}$. 
TABLE 3.1

Results for the computed radii for the matrix A.

\begin{tabular}{|c|c|c|c|c|c|c|c|}
\hline$i$ & 1 & 2 & 3 & 4 & 5 & 6 & 7 \\
\hline$r_{i}(A)$ & 456 & 313 & 317 & 854 & 807 & 807 & 540 \\
\hline$s_{i}(A)$ & 409.93 & 222.92 & 226.38 & 494.37 & 634.08 & 634.08 & 304.20 \\
\hline
\end{tabular}
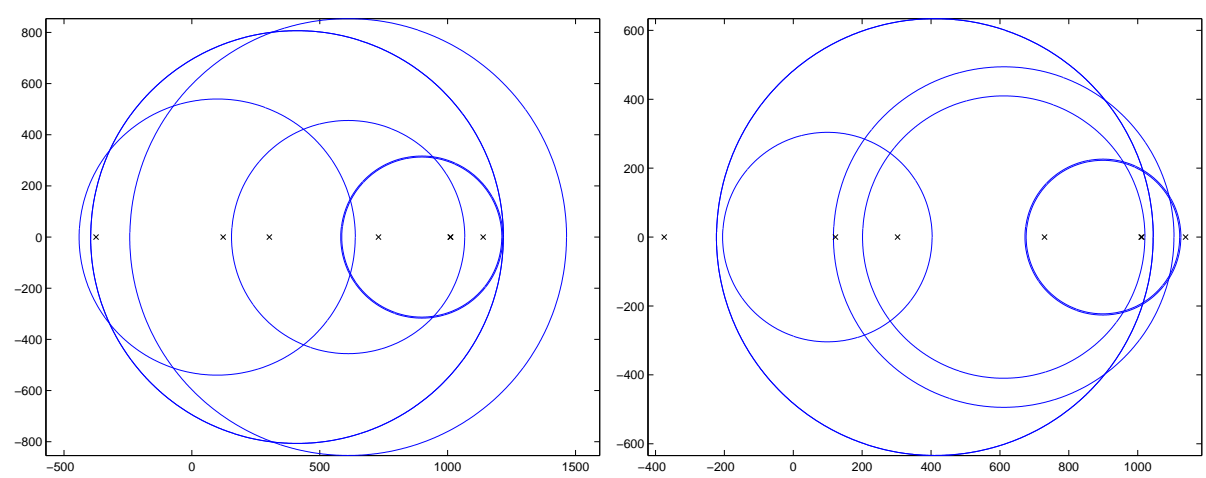

Picture 1: Gershgorin's discs $G_{i}$ (on the left) and the Euclidean discs $E_{i}$ (on the right).

Notice that some eigenvalues of $A$ are outside the set $\bigcup_{i=1}^{n} E_{i}$.

EXAMPLE 3.2. We generated random matrices of entries from the distribution $N(0,1)$ from Matlab's function "randn". We use the following Matlab's code to produce the matrix $A(20 \times 20)$, which is unitary in floating point arithmetic.
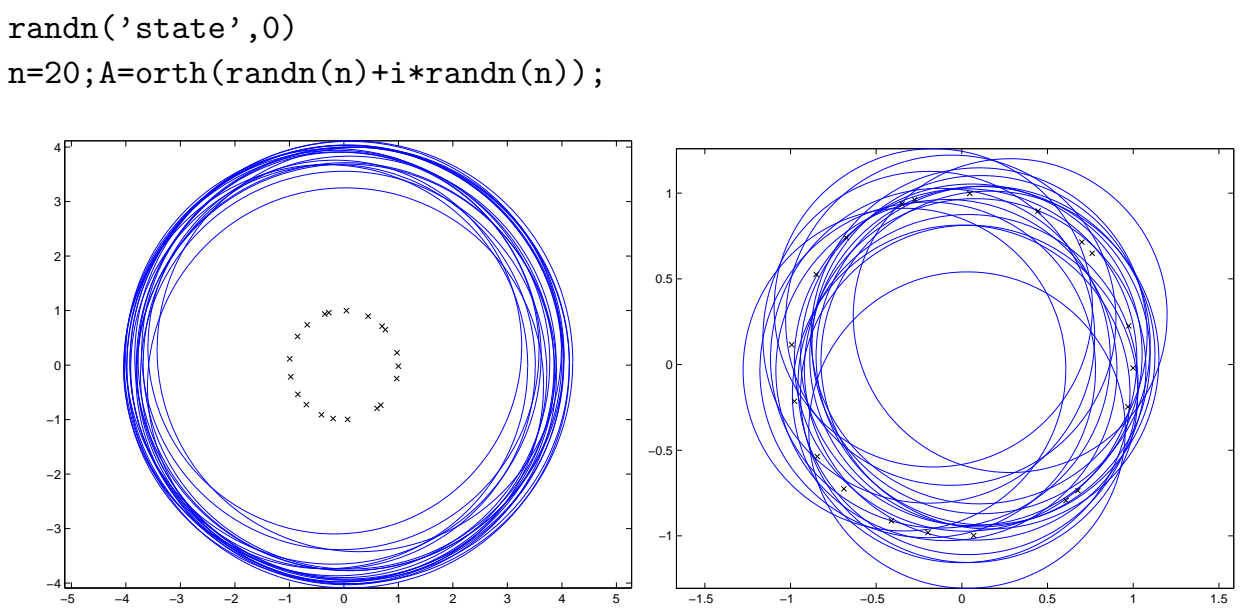

Picture 2: Gershgorin's discs $G_{i}$ (on the left) and the Euclidean discs $E_{i}$ (on the right). 


\section{REFERENCES}

[1] L. Cvetković, V. Kostić, and R. Varga. A new Geršgorin-type eigenvalue inclusion set. Electron. Trans. Numer. Anal., 18:73-80, 2004.

[2] E. Deutsch. Matricial norms. Numer. Math., 16:73-84, 1970.

[3] D.G. Feingold and R.S. Varga. Block diagonally dominant matrices and generalizations of the Gerschgorin circle theorem. Pacific J.Math., 12:1241-1250, 1962.

[4] G. Hämmerlin and Karl-Heinz Hoffmann. Numerical Mathematics. Springer-Verlag, 1991.

[5] D. Meyer and K. Veselić. On some new inclusion theorems for the eigenvalues of partitioned matrices. Numer. Math., 34:431-437, 1980.

[6] A.M. Ostrowski. On some metrical properties of operator matrices and matrices partitioned into blocks. J. Math. Anal. Appl., 2:161-209, 1961.

[7] V. Pták and J. Zemánek. Continuité Lipschitzienne du spectre comme fonction d'un opérateur normal. Commentationes Mathematicae Universitatis Carolinae, 17(3):507-512, 1976.

[8] J.F. Queiró and A.L. Duarte. Imbedding conditions for normal matrices. Linear Algebra Appl., 430:1806-1811, 2009.

[9] A. Smoktunowicz. An inequality involving diagonal elements and eigenvalues. Solution 23-5.4. IMAGE: The Bulletin of the International Linear Algebra Society, 24:11-13, 2000.

[10] A. Smoktunowicz. Block matrices and symmetric perturbations. Linear Algebra Appl., 429:2628-2635, 2008.

[11] R.S. Varga. Geršgorin and His Circles. Springer-Verlag, Berlin, 2004. 